\title{
Pseudoprogression in a Patient With Glioblastoma Presenting as a Neurosurgical Emergency
}

\author{
Wajid M.H. Sayeed, Jacob C. Easaw, Amitabh D. Singh
}

Keywords: chemotherapy, glioblastoma, glioma, neuro-oncology, pseudoprogression, radiotherapy, temozolomide, uncal herniation

doi:10.1017/cjn.2015.53

Can J Neurol Sci. 2015; 42: 347-349

Glioblastoma (GBM) is an aggressive primary intracranial neoplasm that, if left untreated, carries a median survival of about 10 weeks. Following the landmark publication by Stupp et al in $2005,{ }^{1}$ the standard of care for patients with newly diagnosed GBM has been 6 weeks of radiotherapy (in fractions of 2 Gy for a total of 60 Gy), with concurrent daily low-dose temozolomide (TMZ) (dosed at $75 \mathrm{mg} / \mathrm{m}^{2}$ daily) followed by up to 12 cycles of adjuvant TMZ (five of every 28 days, dosed at $150-200 \mathrm{mg} / \mathrm{m}^{2} /$ day). Most centres follow patients receiving this therapy with MRI every 8-12 weeks to assess disease status.

Combined TMZ and radiation are known to cause increased contrast enhancement in the first postchemoradiotherapy MRI in nearly half of all patients. ${ }^{2}$ However, a diagnosis of either pseudoprogression (radiation-induced change) or true disease progression can only be made retrospectively by comparing with another MRI done 2 months later. Pseudoprogression occurs from radiation-induced inflammation characterized by abnormal vessel permeability, causing the contrast material to leak into the brain parenchyma, thus mimicking disease progression. These changes diminish in the MRI done 2 months later. ${ }^{1}$

Previously, tumour response to therapy was assessed by the MacDonald criteria. In 2010, a new set of criteria was published by the Response Assessment in Neuro-Oncology (RANO) Working Group ${ }^{3}$ which precluded the diagnosis of true disease progression within the first 12 weeks of completion of chemoradiotherapy unless either new lesions appear outside the radiation field or histopathology confirms a mitotically active neoplasm. In our previous article, we have shown that clinical deterioration does not help to differentiate between true disease progression and pseudoprogression. ${ }^{4}$

Because a diagnosis of true disease progression often prompts a change in treatment (possible reoperation and different chemotherapy), it is important for oncologists and neurosurgeons alike to recognize this problem-the inability to differentiate pseudoprogression from true disease progression-and not abandon the ongoing chemotherapy plan without a follow-up MRI (done beyond the 12-week period). Consequently, Canadian guidelines recommend that patients continue with a minimum of three cycles of TMZ followed by a repeat MRI in the absence of urgent clinical or radiographic indications for surgical intervention.
From a neurosurgical perspective, surgery done within 12 weeks of completing chemoradiation may be considered in exceptional cases. In our centre, it is general practice not to operate upon patients with possible pseudoprogression because about $50 \%$ of such cases stabilize with further cycles of TMZ. ${ }^{2}$ In this article, we present an important exception to usual practice in which surgery was indicated despite what was later confirmed to be pseudoprogression.

\section{Case Report}

A 48-year-old man presented to the neurooncology service at Tom Baker Cancer Centre, Calgary, following surgery for a right temporal GBM in July 2013. His initial symptoms included severe headaches, inattention, and progressively diminished memory. MRI of his brain revealed a $7.1 \times 3.9 \mathrm{~cm}$ heterogeneously enhancing lesion in the right temporal lobe, for which he underwent surgery (Figure 1). The tumour showed no IDH mutation, and was negative for epidermal growth factor receptor vIII. The test for O6-methylguanine-DNA methyltransferase promoter methylation was indeterminate.

Postoperatively, the patient was treated with 6 weeks of concurrent chemotherapy (TMZ) and radiotherapy (60 Gy) from midAugust to early October 2013. He presented in November 2013 to the clinic complaining of severe, holocranial headaches and fatigue and was treated with intravenous dexamethasone. Oral dexamethasone was thereafter increased to $4 \mathrm{mg}$ three times daily. Because his functional status was suboptimal at that time, adjuvant chemotherapy was deferred.

Two weeks later, the patient was reevaluated for initiating adjuvant TMZ. In the interim, however, he had deteriorated clinically with worsening fatigue, nausea, confusion, and declining memory despite being on high-dose steroids. Physical examination revealed a drowsy patient who was giving intermittent responses to command. Right-sided ptosis and left pronator drift were noted, which were not present earlier.

An emergent MRI of the brain revealed enlargement of an enhancing lesion within the field of radiation, resulting in uncal herniation causing compression and torsion of the midbrain (Figure 2).

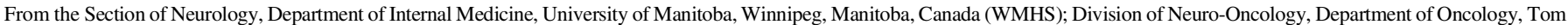
Baker Cancer Centre, Calgary, Alberta, Canada (JCE, ADS).

Received June 10, 2014. Final Revisions Submitted February 11, 2015.

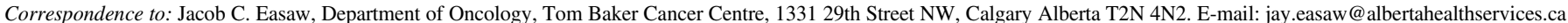




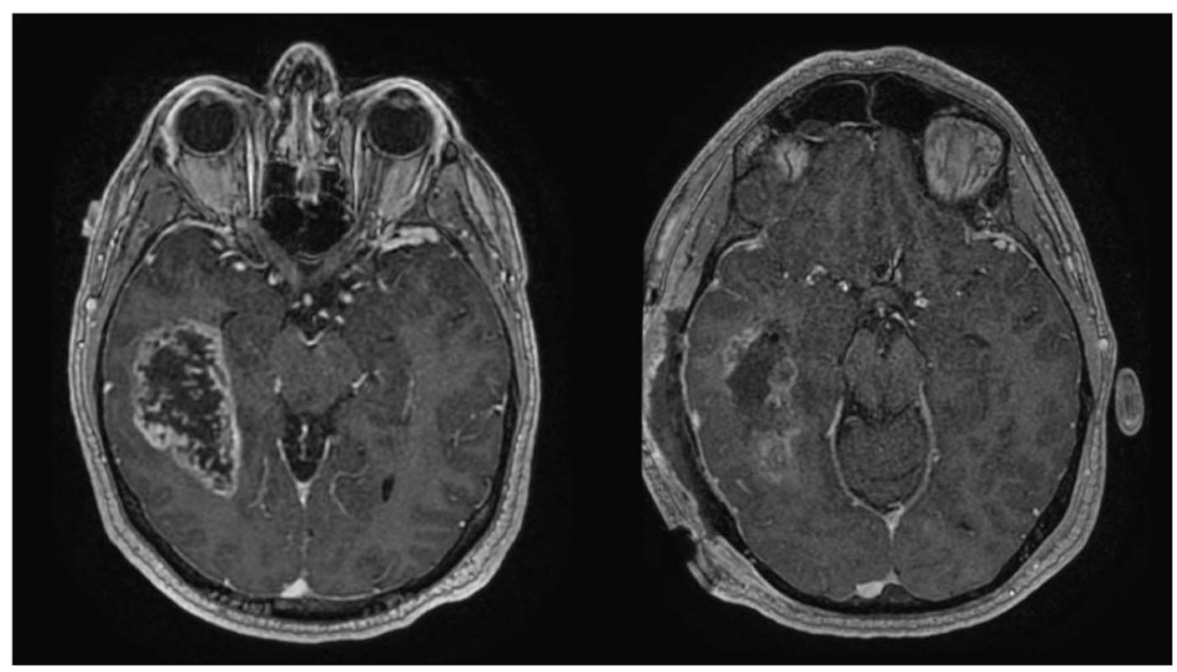

Figure 1: Left: preoperative T1 gadolinium-enhanced MRI demonstrating $7.1 \times 3.9 \mathrm{~cm}$ right temporal enhancing lesion with associated mass effect. Right: postoperative T1 gadoliniumenhanced MRI showing reduction in mass effect.

Although the MRI was done within 12 weeks of completing chemoradiation (when approximately $50 \%$ of patients with increasing contrast enhancing areas have pseudoprogression), his surgeon was alerted to the possibility of the neurosurgical emergency. He was admitted and underwent a surgical decompression in late November 2013. Histopathology of excised tissue showed glioblastoma of moderate cellularity with a low proliferative rate (no mitotic figures, low Ki67), and microvasculature demonstrating thickened, hyalinized walls with rare examples of fibrinoid necrosis. This was consistent with postradiation glioblastoma. The extent of decompression was inadequate, and the patient's neurological condition worsened, necessitating another surgery (anterior temporal lobectomy). His clinical condition improved thereafter and he was started on adjuvant
TMZ (5/28 day schedule). The patient went on to survive an additional 5 months and then died in March 2014.

\section{Discussion}

This case provides an example of an absolute indication for surgical decompression to address a life-threatening condition, despite it being due to the typically nonmalignant phenomenon of pseudoprogression.

Pseudoprogression poses significant challenges for management because it is a diagnosis that can only be made retrospectively. Although some authors have used the presence of characteristics such as decreased cellularity and fibrinoid necrosis to define pseudoprogression on biopsy, no universally agreed-upon

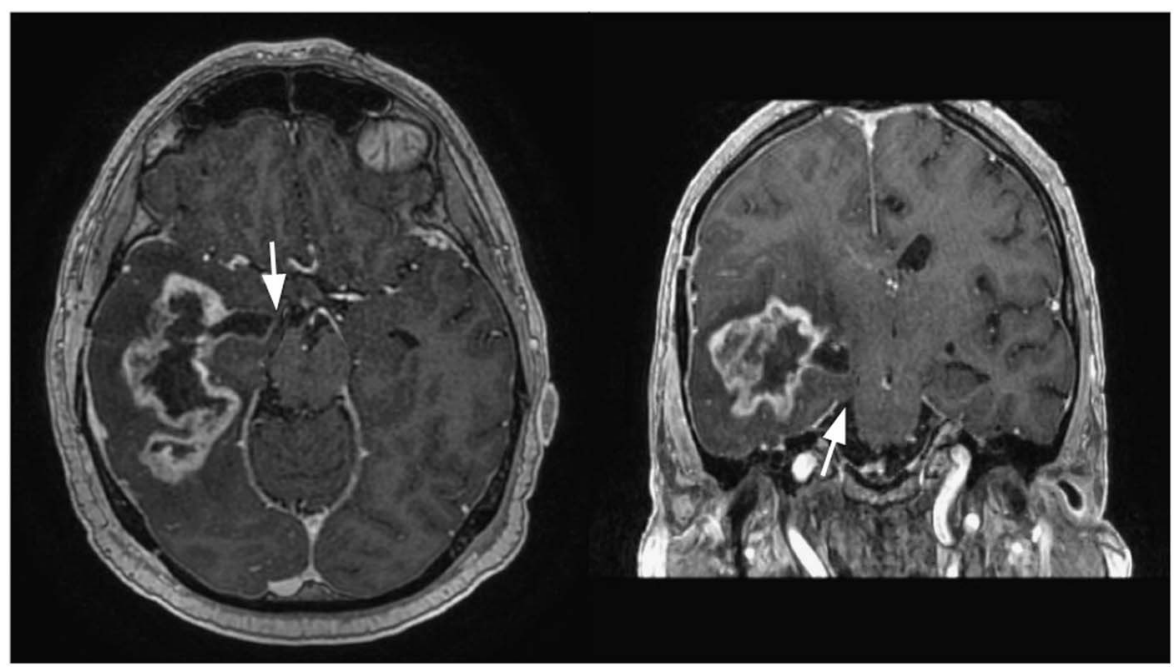

Figure 2: T1 gadolinium-enhanced MRI (done 7 weeks after chemoradiation) demonstrating markedly increased contrast enhancement (as compared with postoperative MRI in Figure 1 [right]) causing uncal herniation. Axial (left) and coronal (right) images show midbrain compression and herniation of the uncus into the suprasellar cistern, respectively. 
Table 1: Summary of RANO criteria

\begin{tabular}{|c|c|c|c|c|}
\hline Criterion & $\mathbf{C R}$ & PR & SD & PD \\
\hline T1 gadolinium-enhancing lesions & None & $\geq 50 \%$ decreased & $<50 \%$ decreased but $<25 \%$ increased & $\geq 25 \%$ increased \\
\hline T2/FLAIR & Stable or decreased & Stable or decreased & Stable or decreased & Increased \\
\hline New lesion & None & None & None & Present \\
\hline Corticosteroids & None & Stable or decreased & Stable or decreased & NA \\
\hline Clinical status & Stable or improved & Stable or improved & Stable or improved & Worsened \\
\hline Requirement for classification & All & All & All & Any \\
\hline
\end{tabular}

Adapted from Wen et al. ${ }^{3}$

$\mathrm{CR}$, complete response; PR, partial response; SD, stable disease; PD, progressive disease; FLAIR, fluid attenuated inversion recovery.

histopathological definition of pseudoprogression exists, and features seen at biopsy frequently overlap early radiation-induced necrotic changes. ${ }^{2,4}$ Ultimately, the diagnosis is confirmed on clinical and radiographic grounds.

Before the publication of the RANO criteria in 2010, the MacDonald criteria were commonly used in clinical trials to judge response to treatment. One of their major limitations, and thus one of the reasons for their revision, was overdiagnosis of disease progression, leading overaccrual and false-positive results in clinical trials for recurrent glioblastoma. ${ }^{3}$ The RANO criteria divide tumour response into four categories: complete response, partial response, stable disease, and progressive disease (Table 1). Importantly, the RANO criteria emphasize that true disease progression cannot be diagnosed on MRI alone within 12 weeks of chemoradiotherapy, barring new lesions beyond the $80 \%$ (radiation) isodose line. The revised criteria do not imply that pseudoprogression is itself harmless, and as our case demonstrates, it can have life-threatening consequences if not recognized.

In the cohort published by Roldan and coworkers, the incidence of early pseudoprogression (occurring within 2 months of completing chemoradiotherapy) was $50 \%$ in patients with GBM. ${ }^{2}$ Our patient had presented with worsening of his neurological condition and ipsilateral ptosis (indicative of partial third nerve paresis). His MRI was done 7 weeks after completing chemoradiotherapy. By convention, he would have been treated by an increase in steroids and surgery (or a change in chemotherapy) would have been deferred until a second MRI showed further increase in the enhancing areas. Had such an approach been taken, the patient would have likely suffered catastrophic uncal herniation and resultant midbrain compression.

Although there is no standard of care for imaging to distinguish pseudoprogression from true progression, several strategies are under investigation for noninvasive assessment of potentially worrisome lesions. Conventional MRI features such as new callosal involvement and new subependymal enhancement can be indicative of true progression, but its utility is otherwise limited. Other MRI-based modalities as well as positron emission tomography tracers such as ${ }^{18} \mathrm{~F}$-deoxyglucose, $O-\left(2-{ }^{18} \mathrm{~F}\right.$-fluoroethyl)-1tyrosine, ${ }^{18} \mathrm{~F}$-dopamine, ${ }^{11} \mathrm{C}$-methionine, and ${ }^{13} \mathrm{~N}-\mathrm{NH}_{3}$ have been recently tested. ${ }^{5}$ Although no single modality has been shown to be uniformly successful in differentiating between pseudoprogression and true progression, the combination of multiple modalities may hold the key to the creation of a useful imaging approach to diagnosis.

It is important for oncologists to recognize imminent neurosurgical emergencies in patients with possible pseudoprogression and arrange for an urgent surgical consultation. Likewise, it is important for neurosurgeons to be aware of the phenomenon and significance of pseudoprogression that occurs in about half the patients undergoing chemoradiation and is conventionally seen during the first 12 weeks after completing chemoradiation. ${ }^{2}$ Because there are no radiological or clinical criteria to distinguish between true disease progression and pseudoprogression during this period, surgery may be deferred in a majority of patients until a subsequent MRI shows further increase in contrast enhancing areas, suggestive of true disease progression. The authors urge readers to assess both the MRI and the clinical status of patients during the first 12 weeks postchemoradiation with extreme care as a means to identify patients in need of urgent surgical decompression, despite the possibility of pseudoprogression being present.

\section{DisClosures}

WMHS, JE, and AS have no disclosures to report.

\section{REFERENCES}

1. Stupp R, Mason WP, van den Bent MJ, et al. Radiotherapy plus concomitant and adjuvant temozolomide for glioblastoma. N Engl J Med. 2005;10:987-96.

2. Roldán GB, Scott JN, McIntyre JB, et al. Population-based study of pseudoprogression after chemoradiotherapy in GBM. Can J Neurol Sci. 2009;36:617-22.

3. Wen PY, Macdonald DR, Reardon DA, et al. Updated response assessment criteria for high-grade gliomas: response assessment in neuro-oncology working group. J Clin Oncol. 2010;28: 1963-72.

4. Singh AD, Easaw JC. Does neurologic deterioration help to differentiate between pseudoprogression and true disease progression in newly diagnosed glioblastoma multiforme? Curr Oncol. 2012;19:e295-8.

5. O'Brien BJ, Colen RR. Post-treatment imaging changes in primary brain tumors. Curr Oncol Rep. 2014;16:397. 